\title{
COMPUTATION OF THE PERIMETER OF MEASURABLE SETS VIA THEIR COVARIOGRAM. APPLICATIONS TO RANDOM SETS
}

\author{
BRUNO GALERNE
}

CMLA, ENS Cachan, CNRS, UniverSud, 61 Avenue du Président Wilson, F-94230 Cachan e-mail: galerne@cmla.ens-cachan.fr

(Accepted February 16, 2011)

\begin{abstract}
The covariogram of a measurable set $A \subset \mathbb{R}^{d}$ is the function $g_{A}$ which to each $y \in \mathbb{R}^{d}$ associates the Lebesgue measure of $A \cap(y+A)$. This paper proves two formulas. The first equates the directional derivatives at the origin of $g_{A}$ to the directional variations of $A$. The second equates the average directional derivative at the origin of $g_{A}$ to the perimeter of $A$. These formulas, previously known with restrictions, are proved for any measurable set. As a by-product, it is proved that the covariogram of a set $A$ is Lipschitz if and only if $A$ has finite perimeter, the Lipschitz constant being half the maximal directional variation. The two formulas have counterparts for mean covariogram of random sets. They also permit to compute the expected perimeter per unit volume of any stationary random closed set. As an illustration, the expected perimeter per unit volume of stationary Boolean models having any grain distribution is computed.
\end{abstract}

Keywords: Boolean model, covariogram, directional variation, random closed sets, set of finite perimeter, specific variation.

\section{INTRODUCTION}

The object of study of this paper is the covariogram $g_{A}$ of a measurable set $A \subset \mathbb{R}^{d}$ defined for all $y \in \mathbb{R}^{d}$ by $g_{A}(y)=\mathscr{L}^{d}(A \cap(y+A))$, where $\mathscr{L}^{d}$ denotes the Lebesgue measure. Note that some authors prefer the terms set covariance or covariance function (Cabo and Janssen, 1994; Cabo and Baddeley, 1995; Rataj, 2004).

Given the covariogram $g_{A}$ of an unknown set $A$, a general inverse problem is to determine the geometric information on $A$ that $g_{A}$ contains. As an important example, Averkov and Bianchi have recently established Matheron's conjecture: up to a translation and a reflection, convex bodies of $\mathbb{R}^{2}$, that is compact convex sets with non-empty interior, are fully determined by their covariogram (see (Averkov and Bianchi, 2009) and the references within). Contrary to the above mentioned result, this paper focuses on geometric information which is shown to be contained in the covariogram of any measurable set: the perimeter.

As our main results will demonstrate, the perimeter which can be computed from the covariogram is the one from the theory of functions of bounded variation (Ambrosio et al., 2000). In this framework, the perimeter of a set $A$ is defined by

$$
\begin{aligned}
\operatorname{Per}(A)=\sup \left\{\int_{\mathbb{R}^{d}} \mathbb{1}_{A}(x) \operatorname{div} \varphi(x) \mathrm{d} x:\right. \\
\left.\varphi \in \mathscr{C}_{c}^{1}\left(\mathbb{R}^{d}, \mathbb{R}^{d}\right),\|\varphi\|_{\infty} \leq 1\right\},
\end{aligned}
$$

and the directional variation in the direction $u \in S^{d-1}$ of $A$ is (Ambrosio et al., 2000, Section 3.11)

$$
\begin{aligned}
V_{u}(A)=\sup \left\{\int_{\mathbb{R}^{d}} \mathbb{1}_{A}(x)\langle\nabla \varphi(x), u\rangle \mathrm{d} x:\right. \\
\left.\varphi \in \mathscr{C}_{c}^{1}\left(\mathbb{R}^{d}, \mathbb{R}\right),\|\varphi\|_{\infty} \leq 1\right\},
\end{aligned}
$$

where $\mathscr{C}_{c}^{1}\left(\mathbb{R}^{d}, \cdot\right)$ denotes the set of continuously differentiable functions with compact support. The non-specialist reader may ask how the perimeter $\operatorname{Per}(A)$ is related to the $(d-1)$-Hausdorff measure $\mathscr{H}^{d-1}$ of the topological boundary $\partial A$, which one might consider to be the intuitive notion of surface area. Let us recall that if $A$ is a compact set with Lipschitz boundary (e.g., $A$ is a convex body), then $\operatorname{Per}(A)=\mathscr{H}^{d-1}(\partial A)$, whereas in the general case we only have $\operatorname{Per}(A) \leq \mathscr{H}^{d-1}(\partial A)$ (Ambrosio et al., 2000, Proposition 3.62). More precisely, if one defines the essential boundary $\partial_{e} A$ of $A$ as the set of points of $\mathbb{R}^{d}$ which are neither Lebesgue density points of $A$ nor of the complementary of $A$, then $\partial_{e} A \subset \partial A$ and $\operatorname{Per}(A)=\mathscr{H}^{d-1}\left(\partial_{e} A\right) \leq \mathscr{H}^{d-1}(\partial A)$ (Ambrosio et al., 2000, Eq. 3.62). As shown in Chlebík (1997), the same conclusion holds for directional variations: defining the projection measure $\mu_{u}$ in the direction $u \in S^{d-1}$ by

$$
\mu_{u}(B)=\int_{(\mathbb{R} u)^{\perp}} \mathscr{H}^{0}(B \cap(x+\mathbb{R} u)) \mathscr{H}^{d-1}(\mathrm{~d} x),
$$

for all measurable subsets $B \subset \mathbb{R}^{d}$, one has $V_{u}(A)=$ $\mu_{u}\left(\partial_{e} A\right) \leq \mu_{u}(\partial A)$. In particular, if $A$ is a convex body then $V_{u}(\bar{A})=2 \mathscr{H}^{d-1}\left(p_{u}(A)\right)$, where $p_{u}$ denotes the orthogonal projection with direction $u$. 
Results. We prove that for every measurable set $A$ of finite Lebesgue measure,

$$
\lim _{r \rightarrow 0} \frac{g_{A}(0)-g_{A}(r u)}{|r|}=\frac{1}{2} V_{u}(A), u \in S^{d-1} .
$$

In addition, noting $\left(g_{A}^{u}\right)^{\prime}(0+):=\lim _{r \rightarrow 0+} \frac{g_{A}(r u)-g_{A}(0)}{r}$ the right directional derivatives at the origin of the covariogram, it is shown that

$$
\operatorname{Per}(A)=-\frac{1}{\omega_{d-1}} \int_{S^{d-1}}\left(g_{A}^{u}\right)^{\prime}(0+) \mathscr{H}^{d-1}(\mathrm{~d} u),
$$

where $\omega_{d-1}$ denotes the Lebesgue measure of the unit ball in $\mathbb{R}^{d-1}$. Hence, for any measurable set $A$, the perimeter $\operatorname{Per}(A)$ can be computed from the directional derivatives at the origin of the covariogram $g_{A}$. As a by-product, it is also shown that a measurable set $A$ has finite perimeter if and only if its covariogram $g_{A}$ is Lipschitz, and in this case the Lipschitz constant is given by

$$
\operatorname{Lip}\left(g_{A}\right)=\frac{1}{2} \sup _{u \in S^{d-1}} V_{u}(A)
$$

Previous work. Eq. 1 has already been proved for certain classes of sets. It was well-known by the mathematical morphology school (Matheron, 1965; Haas et al., 1967; Matheron, 1975; 1986) that the right directional derivative at the origin of the covariogram $g_{A}$ of a convex body equals minus the surface area of the orthogonal projection of the set $A$. The convexity assumption was relaxed in (Rataj, 2004) where Rataj extends the result to compact sets in $\mathscr{U}_{P R}$ satisfying a condition of full-dimensionality, $\mathscr{U}_{P R}$ being the family of locally finite unions of sets with positive reach such that all their finite intersections also have positive reach $^{1}$. In this more general framework, the surface area of the orthogonal projection is replaced by the total projection $T P_{u}(A)$ of $A$, the directional analogue of the $(d-1)$-total curvature $\Phi_{d-1}(A)$ of $A$ (Federer, 1959). Eq. 1 thus implies that if $A$ is a full-dimensional compact $\mathscr{U}_{P R}$-set then $V_{u}(A)=2 T P_{u}(A)$. This identity is the directional equivalent of a recent result due to Ambrosio, Colesanti, and Villa (2008): a fulldimensional compact set with positive reach $A$ satisfies $\operatorname{Per}(A)=2 \Phi_{d-1}(A)$ (Ambrosio et al., 2008, Theorem 9) (one could directly prove that $V_{u}(A)=2 T P_{u}(A)$ by using the techniques developed in (Ambrosio et al., 2008) and (Rataj, 2004)). Since Eq. 1 is valid for any measurable set $A$ such that $\mathscr{L}^{d}(A)<+\infty$, one can argue that the directional variation is the relevant general concept when it comes to the derivative at the origin of the covariogram.
Eq. 2 has been widely stated in the mathematical morphology literature (Haas et al., 1967; Matheron, 1975; Serra, 1982; Lantuéjoul, 2002), under (more or less explicit) regularity assumptions on the set $A$. We rigorously show that it is valid for any measurable set $A$ having finite Lebesgue measure, provided the perimeter $\operatorname{Per}(A)$ is understood as the variation of $A$.

The Lipschitz continuity of the covariogram seems to have received less attention in the literature. It is stated in (Matheron, 1986) that the covariogram of a compact convex set is Lipschitz and the upper bound of the Lipschitz constant given by Matheron is twice the actual value of this constant.

Applications. The covariogram is of particular importance in stochastic geometry when dealing with random closed sets (RACS) (Matheron, 1975; Stoyan et al., 1995; Molchanov, 2005; Schneider and Weil, 2008). In this context, one defines the mean covariogram of a RACS $X$ as the function $\gamma_{X}(y)=$ $\mathbb{E}\left(\mathscr{L}^{d}(X \cap(y+X))\right)$. The mean covariogram of a RACS $X$ is related to the probability that two given points belong to $X$ according to the following relation

$$
\gamma_{X}(y)=\int_{\mathbb{R}^{d}} \mathbb{P}(x \in X \text { and } x+y \in X) \mathrm{d} x .
$$

As a consequence the mean covariogram is systematically involved in second order statistics of classic germ-grain models, such as the Boolean model (Matheron, 1975; Stoyan et al., 1995; Schneider and Weil, 2008), the shot noise model (Rice, 1977; Heinrich and Schmidt, 1985), or the dead leaves model (Matheron, 1968; Jeulin, 1997; Lantuéjoul, 2002; Bordenave et al., 2006).

All the established properties of covariograms of deterministic sets extend to the case of mean covariograms of random closed sets. In particular, the stochastic equivalent of Eqs. 1 and 2 show that the expectations of the variations of a RACS $X$ are proportional to the directional derivatives of its mean covariogram $\gamma_{X}$ (see property 8 of Proposition 16).

If $X$ is any stationary RACS, then its mean covariogram only takes values in $\{0,+\infty\}$ and thus is always degenerate. Nevertheless Eqs. 1 and 2 also permit to study the mean variation of stationary RACS. Define the specific directional variation $\theta_{V_{u}}(X)$ of $X$ as the mean amount of directional variation of $X$ per unit volume (see Section "Specific variation of a stationary RACS" for a detailed definition). For any stationary RACS $X$, it is shown using Eq. 1 that

$$
\theta_{V_{u}}(X)=2 \lim _{r \rightarrow 0} \frac{1}{|r|} \mathbb{P}(r u \in X, 0 \notin X) .
$$

\footnotetext{
${ }^{1}$ We refer to (Federer, 1959) and (Rataj and Zähle, 2001) for definitions and results regarding sets with positive reach and $\mathscr{U}_{P R}$-sets respectively.
} 
Again, integrating over all directions $u$, one obtains an expression of the specific variation $\theta_{V}(X)$ of $X$ (i.e., the mean amount of variation of $X$ per unit volume)

$$
\begin{aligned}
& \theta_{V}(X) \\
& =\frac{1}{\omega_{d-1}} \int_{S^{d-1}} \lim _{r \rightarrow 0} \frac{1}{|r|} \mathbb{P}(r u \in X, 0 \notin X) \mathscr{H}^{d-1}(\mathrm{~d} u) .
\end{aligned}
$$

As for Eq. 2, the above formula has been stated in the early works of Matheron (Matheron, 1967, p. 30) (Lantuéjoul, 2002, p. 26), but assumptions on the regularity of the RACS were not clearly formulated. It should be emphasized that the specific variation is well-defined for any stationary RACS, and that it can be easily computed as soon as one knows the probabilities $\mathbb{P}(r u \in X, 0 \notin X)$. As an illustration, the specific directional variations and the specific variation of stationary Boolean models are computed in this paper. The obtained expressions generalize known statistics of Boolean models with convex grains (Schneider and Weil, 2008). Because it is well-defined for any stationary RACS and easily computable, we claim that, when dealing with non negligible RACS, the specific variation is an interesting alternative to other extension of the usual specific surface area that derives from Steiner's formula (Schneider and Weil, 2008).

Contents. In Section "Covariogram of a measurable set" the covariogram $g_{A}$ of a Lebesgue measurable set $A$ is defined and several properties of $g_{A}$ are recalled and established. In particular it is shown that as soon as $A$ is non negligible its covariogram $g_{A}$ is a strictly positive-definite function. The following section gathers several known results from the theory of functions of bounded directional variation. In Section "Directional variation, perimeter and covariogram of measurable sets", the main results relating both the derivative at the origin and the Lipschitz continuity of the covariogram of a set to its directional variations and its perimeter are stated. Finally, in the last section, applications of these results to the theory of random closed sets are discussed and illustrated.

\section{COVARIOGRAM OF A MEASURABLE SET}

Definition 1 (Covariogram of a measurable set). Let $A \subset \mathbb{R}^{d}$ be a $\mathscr{L}^{d}$-measurable set of finite Lebesgue measure. The covariogram of $A$ is the function $g_{A}$ : $\mathbb{R}^{d} \rightarrow[0,+\infty)$ defined for all $y \in \mathbb{R}^{d}$ by

$$
g_{A}(y)=\mathscr{L}^{d}(A \cap(y+A))=\int_{\mathbb{R}^{d}} \mathbb{1}_{A}(x) \mathbb{1}_{A}(x-y) \mathrm{d} x .
$$

As initially noted by Matheron (1965), the covariogram of $A$ can be expressed as the convolution of the indicator functions of $A$ and its symmetric $\check{A}=$ $\{-x \mid x \in A\}$ :

$$
g_{A}=\mathbb{1}_{A} * \mathbb{1}_{\check{A}} .
$$

As illustrated in the following proposition, this point of view is useful to establish some analytic properties of $g_{A}$.

Proposition 2. Let $A \subset \mathbb{R}^{d}$ be a $\mathscr{L}^{d}$-measurable set of finite Lebesgue measure and $g_{A}$ be its covariogram. Then

1. For all $y \in \mathbb{R}^{d}, 0 \leq g_{A}(y) \leq g_{A}(0)=\mathscr{L}^{d}(A)$.

2. $g_{A}$ is even: for all $y \in \mathbb{R}^{d}, g_{A}(-y)=g_{A}(y)$.

3. $\int_{\mathbb{R}^{d}} g_{A}(y) \mathrm{d} y=\mathscr{L}^{d}(A)^{2}$.

4. $g_{A}$ is uniformly continuous over $\mathbb{R}^{d}$ and $\lim _{|y| \rightarrow+\infty} g_{A}(y)=0$.

Proof. The proofs of the three first properties are straightforward. Since $\mathbb{1}_{A}$ and $\mathbb{1}_{\check{A}}$ are in $L^{2}\left(\mathbb{R}^{d}\right)$, the fourth property is obtained in applying the $L^{p}$ $L^{p^{\prime}}$-convolution theorem to $g_{A}=\mathbb{1}_{A} * \mathbb{1}_{\check{A}}$ (see Hirsch and Lacombe, 1999, Proposition 3.2 p. 171, for example).

It is well-known that the covariogram is a positivedefinite function (Matheron, 1965, p. 22; Lantuéjoul, 2002 , p. 23). The next proposition improves slightly this result. In particular, it shows that for all $x \neq 0$, $g_{A}(x)<g_{A}(0)$.

Proposition 3 (Strict positive-definiteness of the covariogram). Let A be a $\mathscr{L}^{d}$-measurable set such that $0<\mathscr{L}^{d}(A)<+\infty$. Then its covariogram $g_{A}$ is a strictly positive-definite function, that is, for all integers $p \geq 1$, for all p-tuples $\left(y_{1}, \ldots, y_{p}\right)$ of distinct vectors of $\mathbb{R}^{d}$, and for all $\left(w_{1}, \ldots, w_{p}\right) \in \mathbb{R}^{p} \backslash\{0\}$ we have

$$
\sum_{j, k=1}^{p} w_{j} w_{k} g_{A}\left(y_{k}-y_{j}\right)>0 .
$$

The proof of Proposition 3 makes use of the following lemma.

Lemma 4 (The translations of an integrable function are linearly independent). Let $f$ be a non null function of $L^{1}\left(\mathbb{R}^{d}\right)$ and let $y_{1}, \ldots, y_{p}$ be $p$ distinct vectors of $\mathbb{R}^{d}$. Then the functions $x \mapsto f\left(x+y_{j}\right), j=1, \ldots, p$, are linearly independent in $L^{1}\left(\mathbb{R}^{d}\right)$. 
Proof. Let $\left(w_{1}, \ldots, w_{p}\right) \in \mathbb{R}^{p}$ be such that

$$
\sum_{j=1}^{p} w_{j} f\left(x+y_{j}\right)=0 \quad \text { for a.e. } x \in \mathbb{R}^{d} .
$$

Applying the Fourier transform we have

$$
\left(\sum_{j=1}^{p} w_{j} e^{i\left\langle\xi, y_{j}\right\rangle}\right) \hat{f}(\xi)=0 \text { for all } \xi \in \mathbb{R}^{d} .
$$

Since $f$ is non null and integrable, $\hat{f}$ is non null and continuous. Hence there exists an open ball $B=B\left(\xi_{0}, r\right)$ of center $\xi_{0} \in \mathbb{R}^{d}$ and radius $r>0$ such that for all $\xi \in B, \hat{f}(\xi) \neq 0$, and thus for all $\xi \in B, S(\xi):=\sum_{j=1}^{p} w_{j} e^{i\left\langle\xi, y_{j}\right\rangle}=0$. One easily shows that the sum $S(\xi)$ is null for all $\xi \in \mathbb{R}^{d}$ in considering the one-dimensional restriction of $S$ on the line containing $\xi$ and $\xi_{0}$ : by the identity theorem, this one-dimensional function is null since it is analytic and null over an open interval. Applying the inverse generalized Fourier transform to $S=0$ shows that $\sum_{j=1}^{p} w_{j} \delta_{y_{j}}=0$. This implies that $w_{1}=\cdots=w_{p}=0$, since by hypothesis the vectors $y_{j}$ are distinct.

Proof of Proposition 3. By Lemma 4, the function $x \mapsto \sum_{j=1}^{p} w_{j} \mathbb{1}_{A}\left(x+y_{j}\right)$ is not a.e. equal to 0 . Hence

$$
\begin{aligned}
& \sum_{j, k=1}^{p} w_{j} w_{k} g_{A}\left(y_{k}-y_{j}\right) \\
& =\sum_{j, k=1}^{p} w_{j} w_{k} \int_{\mathbb{R}^{d}} \mathbb{1}_{A}(x) \mathbb{1}_{A}\left(x-y_{k}+y_{j}\right) \mathrm{d} x \\
& =\sum_{j, k=1}^{p} w_{j} w_{k} \int_{\mathbb{R}^{d}} \mathbb{1}_{A}\left(x+y_{k}\right) \mathbb{1}_{A}\left(x+y_{j}\right) \mathrm{d} x \\
& =\int_{\mathbb{R}^{d}}\left(\sum_{j=1}^{p} w_{j} \mathbb{1}_{A}\left(x+y_{j}\right)\right)^{2} \mathrm{~d} x>0 .
\end{aligned}
$$

Proposition 5. Let $A \subset \mathbb{R}^{d}$ be a $\mathscr{L}^{d}$-measurable set of finite Lebesgue measure and let $g_{A}$ be its covariogram. Then for all $y, z \in \mathbb{R}^{d}$

$$
\left|g_{A}(y)-g_{A}(z)\right| \leq g_{A}(0)-g_{A}(y-z) .
$$

Proof. First let us show that for all measurable sets $A_{1}$, $A_{2}$, and $A_{3}$

$$
\begin{aligned}
& \mathscr{L}^{d}\left(A_{1} \cap A_{2}\right)-\mathscr{L}^{d}\left(A_{1} \cap A_{3}\right) \\
& \leq \mathscr{L}^{d}\left(A_{2} \backslash A_{3}\right)=\mathscr{L}^{d}\left(A_{2}\right)-\mathscr{L}^{d}\left(A_{3} \cap A_{2}\right) .
\end{aligned}
$$

We have

$$
\begin{aligned}
& \mathscr{L}^{d}\left(A_{1} \cap A_{2}\right)-\mathscr{L}^{d}\left(A_{1} \cap A_{3}\right) \\
& \leq \mathscr{L}^{d}\left(A_{1} \cap A_{2}\right)-\mathscr{L}^{d}\left(A_{1} \cap A_{2} \cap A_{3}\right) \\
& =\mathscr{L}^{d}\left(\left(A_{1} \cap A_{2}\right) \backslash\left(A_{1} \cap A_{2} \cap A_{3}\right)\right) .
\end{aligned}
$$

Now using that $\left(A_{1} \cap A_{2}\right) \backslash\left(A_{1} \cap A_{2} \cap A_{3}\right)$ is included in the set $A_{2} \backslash A_{3}$, Eq. 3 is proved. Applying Eq. 3 to the sets $A_{1}=A, A_{2}=y+A$ and $A_{3}=z+A$ we get

$$
\begin{aligned}
g_{A}(y)-g_{A}(z) & =\mathscr{L}^{d}(A \cap(y+A))-\mathscr{L}^{d}(A \cap(z+A)) \\
& \leq \mathscr{L}^{d}(y+A)-\mathscr{L}^{d}((y+A) \cap(z+A)) \\
& =\mathscr{L}^{d}(A)-\mathscr{L}^{d}(A \cap((z-y)+A)) \\
& =g_{A}(0)-g_{A}(z-y) .
\end{aligned}
$$

\section{Remark.}

- The weaker inequality

$$
\left|g_{A}(y)-g_{A}(z)\right| \leq 2\left(g_{A}(0)-g_{A}(y-z)\right)
$$

was established by Matheron (1986, p. 1).

- The inequality of Proposition 5 shows that the Lipschitz continuity of the covariogram only depends on the behavior of the function in 0 .

\section{FACTS FROM THE THEORY OF FUNCTIONS OF BOUNDED DIRECTIONAL VARIATION}

This section gathers necessary results from the theory of functions of bounded variation. For a general treatment of the subject we refer to the textbook of Ambrosio, Fusco, and Pallara (2000). When the enunciated properties of functions of bounded variation are not found in (Ambrosio et al., 2000), full proofs are given for the convenience of the reader. Let us add that these proofs can be skipped without impeding on the understanding of the main results of the paper that will be established in the next section.

For any open subset $U \subset \mathbb{R}^{d}, \mathscr{B}(U)$ denotes the set of Borel subsets of $U$, and we write $V \subset \subset U$ if $V \subset U$ is open and relatively compact in $U$.

Definition 6 (Functions of bounded variation). Let $U$ be an open set of $\mathbb{R}^{d}$. We say that $f \in L_{\text {loc }}^{1}(U)$ is $a$ function of locally bounded variation in $U$ if the distributional derivative of $f$ is representable by a $\mathbb{R}^{d}$ valued Radon measure, i.e., if there exists a $\mathbb{R}^{d}$-valued Radon measure, noted $D f=\left(D_{1} f, \ldots, D_{d} f\right)$, such that for all $\varphi=\left(\varphi_{1}, \ldots, \varphi_{d}\right) \in \mathscr{C}_{c}^{\infty}\left(U, \mathbb{R}^{d}\right)$

$$
\int_{U} f(x) \operatorname{div} \varphi(x) \mathrm{d} x=-\sum_{i=1}^{d} \int_{U} \varphi_{i}(x) D_{i} f(\mathrm{~d} x) .
$$


The vector space of all functions of locally bounded variation in $U$ is denoted by $B V_{\mathrm{loc}}(U)$. The functions $f \in B V_{\text {loc }}(U)$ such that $f \in L^{1}(U)$ and $|D f|(U)<+\infty$ are said to be functions of bounded variation in $U$ and the corresponding function space is denoted by $B V(U)$.

In what follows, $S^{d-1}$ denotes the unit Euclidean sphere in $\mathbb{R}^{d}$. If $\varphi \in \mathscr{C}^{1}(U, \mathbb{R})$ and $u \in S^{d-1}$, we write

$$
\frac{\partial \varphi}{\partial u}(x)=\langle\nabla \varphi(x), u\rangle, x \in \mathbb{R}^{d},
$$

for the directional derivative of $\varphi$ in the direction $u$.

Definition 7 (Functions of bounded directional variation). Let $U$ be an open set of $\mathbb{R}^{d}$ and let $u \in S^{d-1} . \quad f \in L_{\mathrm{loc}}^{1}(U)$ is a function of locally bounded directional variation in the direction $u$ in $U$ if the directional distributional derivative of $f$ in the direction $u$ is representable by a signed Radon measure, i.e., if there exists a signed Radon measure, noted $D_{u} f$, such that for all $\varphi \in \mathscr{C}_{c}^{\infty}(U, \mathbb{R})$

$$
\int_{U} f(x) \frac{\partial \varphi}{\partial u}(x) \mathrm{d} x=-\int_{U} \varphi(x) D_{u} f(\mathrm{~d} x) .
$$

The corresponding space is denoted by $B V_{u, \text { loc }}(U)$. The functions $f \in B V_{u, \mathrm{loc}}(U)$ such that $f \in L^{1}(U)$ and $\left|D_{u} f\right|(U)<+\infty$ are said to be functions of bounded directional variation in the direction $u$ in $U$ and the corresponding function space is denoted by $B V_{u}(U)$.

The variation in $U$ of a function $f \in L_{\mathrm{loc}}^{1}(U)$ is defined by

$$
\begin{aligned}
V(f, U)=\sup \left\{\int_{U} f(x) \operatorname{div} \varphi(x) \mathrm{d} x:\right. \\
\left.\varphi \in \mathscr{C}_{c}^{1}\left(U, \mathbb{R}^{d}\right),\|\varphi\|_{\infty} \leq 1\right\} .
\end{aligned}
$$

A fundamental result of the theory of function of bounded variation states that the variation $V(f, U)$ of $f \in L^{1}(U)$ is finite if and only if $f \in B V(U)$ (Ambrosio et al., 2000). More precisely,

$$
V(f, U)= \begin{cases}|D f|(U) & \text { if } f \in B V(U) \\ +\infty & \text { otherwise }\end{cases}
$$

Similarly, for all $f \in L_{\mathrm{loc}}^{1}(U)$ one defines the directional variation in the direction $u$ of $f$ by

$$
\begin{aligned}
V_{u}(f, U)=\sup \left\{\int_{U} f(x) \frac{\partial \varphi}{\partial u}(x) \mathrm{d} x:\right. \\
\\
\left.\varphi \in \mathscr{C}_{c}^{1}(U, \mathbb{R}),\|\varphi\|_{\infty} \leq 1\right\},
\end{aligned}
$$

and

$$
V_{u}(f, U)= \begin{cases}\left|D_{u} f\right|(U) & \text { if } f \in B V_{u}(U), \\ +\infty & \text { otherwise. }\end{cases}
$$

If $A \subset \mathbb{R}^{d}$ is a $\mathscr{L}^{d}$-measurable set, by definition the perimeter of $A$ in $U$ is the variation of the indicator function $\mathbb{1}_{A}$ in $U$ and one notes $\operatorname{Per}(A, U):=V\left(\mathbb{1}_{A}, U\right)$. Besides, one writes $V_{u}(A, U):=V_{u}\left(\mathbb{1}_{A}, U\right)$ for the directional variation of $A$ in the direction $u$ in $U$. In the case where $U=\mathbb{R}^{d}$, one simply writes $V(f)=$ $V\left(f, \mathbb{R}^{d}\right)$ and $V_{u}(f)=V_{u}\left(f, \mathbb{R}^{d}\right)$, and similarly for the variations of a set.

As shown by the next proposition, given all the directional variations $V_{u}(f, U), u \in S^{d-1}$, one can compute the variation $V(f, U)$.

Proposition 8 (Variation and directional variations). Let $U$ be an open set of $\mathbb{R}^{d}$ and let $f \in L^{1}(U)$. Then, the three following assertions are equivalent:

(i) $f \in B V(U)$.

(ii) $f \in B V_{u}(U)$ for all $u \in S^{d-1}$.

(iii) For all vectors $e_{i}$ of the canonical basis, $f \in B V_{e_{i}}(U)$.

In addition,

$\frac{1}{d} V(f, U) \leq \frac{1}{d} \sum_{i=1}^{d} V_{e_{i}}(f, U) \leq \sup _{u \in S^{d-1}} V_{u}(f, U) \leq V(f, U)$,

and

$$
V(f, U)=\frac{1}{2 \omega_{d-1}} \int_{S^{d-1}} V_{u}(f, U) \mathscr{H}^{d-1}(\mathrm{~d} u),
$$

where $\omega_{d-1}$ denotes the Lebesgue measure of the unit ball in $\mathbb{R}^{d-1}$.

The results of this proposition are mostly from (Chlebík, 1997). A proof is reproduced below for the convenience of the reader. First one needs the following lemma.

Lemma 9. Let $f \in L^{1}(U)$ and let $u_{1}, \ldots, u_{k} \in S^{d-1}$ such that $f \in B V_{u_{j}}(U)$ for all $j=1, \ldots, k$. Then for all $u=\sum_{j} \lambda_{j} u_{j} \in S^{d-1} \cap \operatorname{span}\left\{u_{1}, \ldots, u_{k}\right\}, f \in B V_{u}(U)$.

Proof. With the above notation one easily checks that $D_{u} f:=\sum_{j} \lambda_{j} D_{u_{j}} f$ is a signed Radon measure which represents the directional derivative of $f$ in the direction $u$. Besides, by the triangle inequality $\left|D_{u} f\right|(U) \leq \sum_{j}^{k}\left|\lambda_{j}\right|\left|D_{u_{j}} f\right|(U)<+\infty$, and thus $f \in$ $B V_{u}(U)$. 
Proof of Proposition 8. Clearly, Assertion (ii) implies Assertion (iii). Let us show that (i) implies (ii). Let $f \in B V(U)$, let $D f=\left(D_{1} f, \ldots, D_{d} f\right)$ be the Radon measure representing its distributional derivative, and let $u \in S^{d-1}$. Then $\langle D f, u\rangle:=\sum_{i=1}^{d} u_{i} D_{i} f$ is a signed Radon measure which represents the directional derivative of $f$ in the direction $u$, and by the CauchySchwarz inequality

$$
\begin{aligned}
& V_{u}(f, U)=|\langle D f, u\rangle|(U) \leq \sum_{i=1}^{d}\left|u_{i}\right|\left|D_{i} f\right|(U) \\
& \quad \leq|u||D f|(U)=|D f|(U)=V(f, U)<+\infty .
\end{aligned}
$$

Hence $f \in B V_{u}(U)$ and $V_{u}(f, U) \leq V(f, U)$. Let us now show that (iii) implies $(i)$. For all vectors $e_{i}$ of the canonical basis, $f \in B V_{e_{i}}\left(\mathbb{R}^{d}\right)$ and there exists a signed Radon measure $D_{e_{i}} f$ which represents the distributional partial derivatives of $f$. But then one easily checks that $\left(D_{e_{1}} f, \ldots, D_{e_{d}} f\right)$ is a $\mathbb{R}^{d}$-valued Radon measure which represents the distributional derivative of $f$. In addition, from the definition of the variation

$$
V(f, U) \leq \sum_{i=1}^{d} V_{e_{i}}(f, U)<+\infty
$$

and thus $f \in B V(U)$. This concludes the proof of the announced equivalence as well as the proof of the inequalities since

$$
V(f, U) \leq \sum_{i=1}^{d} V_{e_{i}}(f, U) \leq d \sup _{u \in S^{d-1}} V_{u}(f, U) \leq d V(f, U) .
$$

To finish let us show Eq. 5. First let us suppose that $f \notin B V(U)$ and let us show that the right-hand side of Eq. 5 is equal to $+\infty$. Remark that by Lemma 9 and the equivalence above the set of directions $u \in$ $S^{d-1}$ for which $V_{u}(f, U)<+\infty$ is contained in a linear subspace of dimension less than $d-1$ (otherwise $f$ would be in $B V_{u}(U)$ for all $u$ and consequently $f$ would be in $B V(U)$ ). Hence for $\mathscr{H}^{d-1}$-all $u$ in $S^{d-1}$, $V_{u}(f, U)=+\infty$, and thus the right-hand side of Eq. 5 equals $+\infty$. Let us now suppose that $f \in B V(U)$. By the polar decomposition theorem (Ambrosio et al., 2000, Corollary 1.29) there exists a unique $|D f|$-integrable function $\sigma: U \rightarrow S^{d-1}$ such that $D f=\sigma|D f|$. With this notation, observe that for all $u \in S^{d-1}$ and $A \in$ $\mathscr{B}(U)$,

$$
D_{u} f(A)=\langle D f, u\rangle(A)=\int_{A}\langle\sigma(x), u\rangle|D f|(\mathrm{d} x) .
$$

Hence, by (Ambrosio et al., 2000, Proposition 1.23)

$$
V_{u}(f, U)=\left|D_{u} f\right|(U)=\int_{U}|\langle\sigma(x), u\rangle||D f|(\mathrm{d} x) .
$$

\footnotetext{
${ }^{2}$ L. Ambrosio, personal communication.
}

For all $v \in S^{d-1}$ the following well-known identity holds

$$
\int_{S^{d-1}}|\langle v, u\rangle| \mathscr{H}^{d-1}(\mathrm{~d} u)=2 \omega_{d-1}
$$

Hence by Fubini's theorem

$$
\begin{aligned}
& \int_{S^{d-1}} V_{u}(f, U) \mathscr{H}^{d-1}(\mathrm{~d} u) \\
& =\int_{U}\left(\int_{S^{d-1}}|\langle\sigma(x), u\rangle| \mathscr{H}^{d-1}(\mathrm{~d} u)\right)|D f|(\mathrm{d} x) \\
& =2 \omega_{d-1}|D f|(U) \\
& =2 \omega_{d-1} V(f, U)
\end{aligned}
$$

The next proposition recalls fundamental properties related to the approximation of functions of bounded directional variation. For simplicity we restrict ourselves to the case $U=\mathbb{R}^{d}$. See (Ambrosio et al., 2000, Section 3.11) for the proofs.

Proposition 10 (Directional variation and approximation).

- Variation of smooth functions: If $f \in$ $\mathscr{C}^{1}\left(\mathbb{R}^{d}\right) \cap L^{1}\left(\mathbb{R}^{d}\right)$ then

$$
V_{u}(f)=\int_{\mathbb{R}^{d}}\left|\frac{\partial f}{\partial u}(x)\right| \mathrm{d} x .
$$

- Lower semi-continuity with respect to the $L^{1}$ convergence: If $f_{n}$ converges towards $f$ in $L^{1}\left(\mathbb{R}^{d}\right)$ then $V_{u}(f) \leq \liminf _{n \rightarrow+\infty} V_{u}\left(f_{n}\right)$.

- Approximation by smooth functions: for every function $f \in B V_{u}\left(\mathbb{R}^{d}\right)$, there exists a sequence of smooth functions $f_{n} \in \mathscr{C}^{\infty}\left(\mathbb{R}^{d}\right) \cap B V_{u}\left(\mathbb{R}^{d}\right)$ such that $f_{n}$ converges towards $f$ in $L^{1}\left(\mathbb{R}^{d}\right)$ and $\lim _{n \rightarrow+\infty} V_{u}\left(f_{n}\right)=V_{u}(f)$.

One practical advantage of directional variations $V_{u}(f)$ over the non-directional variation $V(f)$ is that it can be computed from the integrals of difference quotients, as the next proposition recalls. Although this is a standard result of $B V$ functions theory ${ }^{2}$, the author is not aware of any standard textbook which enunciates it. Consequently a proof is given for the convenience of the reader. 
Proposition 11 (Directional variation and difference quotient). Let $u \in S^{d-1}$ and let $f \in L^{1}\left(\mathbb{R}^{d}\right)$ be any integrable function. Then for all $r \neq 0$,

$$
\int_{\mathbb{R}^{d}} \frac{|f(x+r u)-f(x)|}{|r|} \mathrm{d} x \leq V_{u}(f)
$$

and

$$
\lim _{r \rightarrow 0} \int_{\mathbb{R}^{d}} \frac{|f(x+r u)-f(x)|}{|r|} \mathrm{d} x=V_{u}(f) .
$$

Proof. To prove the inequality we can suppose that $f \in$ $B V_{u}\left(\mathbb{R}^{d}\right)$. First suppose that $f \in \mathscr{C}^{1}\left(\mathbb{R}^{d}\right) \cap B V_{u}\left(\mathbb{R}^{d}\right)$. Then

$$
\begin{aligned}
|f(x+r u)-f(x)| & =\left|\int_{0}^{1} r \frac{\partial f}{\partial u}(x+t r u) \mathrm{d} t\right| \\
& \leq \int_{0}^{1}|r|\left|\frac{\partial f}{\partial u}(x+t r u)\right| \mathrm{d} t .
\end{aligned}
$$

Hence, using Fubini's theorem and the first point of Proposition 10,

$$
\begin{aligned}
& \int_{\mathbb{R}^{d}} \frac{|f(x+r u)-f(x)|}{|r|} \mathrm{d} x \\
& \leq \int_{0}^{1}\left(\int_{\mathbb{R}^{d}}\left|\frac{\partial f}{\partial u}(x+t r u)\right| \mathrm{d} x\right) \mathrm{d} t=V_{u}(f) .
\end{aligned}
$$

This inequality is shown to be valid for any $f \in$ $B V_{u}\left(\mathbb{R}^{d}\right)$ by using approximation by smooth functions (see Proposition 10).

Let us now turn to the second part of the statement. Let $f \in L^{1}\left(\mathbb{R}^{d}\right)$. Using the above inequality it is enough to show that

$$
V_{u}(f) \leq \liminf _{r \rightarrow 0} \int_{\mathbb{R}^{d}} \frac{|f(x+r u)-f(x)|}{|r|} \mathrm{d} x .
$$

Let us consider a family of mollifiers $\left(\rho_{\varepsilon}\right)_{\varepsilon>0}$, that is functions $\rho_{\varepsilon}(x):=\varepsilon^{-d} \rho(x / \varepsilon)$ where the function $\rho$ is even, non negative, $\mathscr{C}^{\infty}$, with support contained in the unit ball, and such that $\int_{\mathbb{R}^{d}} \rho(x) \mathrm{d} x=1$. Define $f_{\varepsilon}=$ $f * \rho_{\varepsilon}$. Then $f_{\varepsilon} \in \mathscr{C}^{\infty}\left(\mathbb{R}^{d}\right) \cap L^{1}\left(\mathbb{R}^{d}\right)$ and $f_{\varepsilon}$ converges towards $f$ in $L^{1}\left(\mathbb{R}^{d}\right)$ as $\varepsilon$ tends to 0 . By Fatou's lemma we have

$$
\begin{aligned}
V_{u}\left(f_{\varepsilon}\right) & =\int_{\mathbb{R}^{d}}\left|\frac{\partial f_{\varepsilon}}{\partial u}(x)\right| \mathrm{d} x \\
& \leq \liminf _{r \rightarrow 0} \int_{\mathbb{R}^{d}} \frac{\left|f_{\mathcal{\varepsilon}}(x+r u)-f_{\mathcal{\varepsilon}}(x)\right|}{|r|} \mathrm{d} x .
\end{aligned}
$$

Since

$$
\begin{aligned}
\left\|f_{\mathcal{E}}(\cdot+r u)-f_{\mathcal{E}}(\cdot)\right\|_{1} & =\left\|(f(\cdot+r u)-f(\cdot)) * \rho_{\varepsilon}\right\|_{1} \\
& \leq\|f(\cdot+r u)-f(\cdot)\|_{1}
\end{aligned}
$$

we deduce that for all $\varepsilon>0$

$$
V_{u}\left(f_{\varepsilon}\right) \leq \liminf _{r \rightarrow 0} \int_{\mathbb{R}^{d}} \frac{|f(x+r u)-f(x)|}{|r|} \mathrm{d} x .
$$

Using the lower semi-continuity of the directional variation with respect to the $L^{1}$-convergence we get the result.

\section{DIRECTIONAL VARIATION, PERIMETER AND COVARIOGRAM OF MEASURABLE SETS}

In this section, the main results of the paper are established (see Theorem 13 and Theorem 14).

Lemma 12 ((Matheron, 1986)). Let $A$ be a $\mathscr{L}^{d}$. measurable set having finite Lebesgue measure and let $g_{A}$ be its covariogram. Then for all $y \in \mathbb{R}^{d}$

$$
g_{A}(0)-g_{A}(y)=\frac{1}{2} \int_{\mathbb{R}^{d}}\left|\mathbb{1}_{A}(x+y)-\mathbb{1}_{A}(x)\right| \mathrm{d} x .
$$

Proof.

$$
\begin{aligned}
& \int_{\mathbb{R}^{d}}\left|\mathbb{1}_{A}(x+y)-\mathbb{1}_{A}(x)\right| \mathrm{d} x \\
& =\int_{\mathbb{R}^{d}}\left(\mathbb{1}_{A}(x+y)-\mathbb{1}_{A}(x)\right)^{2} \mathrm{~d} x \\
& =2\left(g_{A}(0)-g_{A}(y)\right) .
\end{aligned}
$$

The identity of Lemma 12, which is due to Matheron (1986), is the key point to apply the results from the theory of functions of bounded directional variations enunciated in the previous section. First, one establishes Eq. 1 and obtains a characterization of sets of finite directional variation.

Theorem 13 (Directional variation and covariogram of measurable sets). Let $A$ be a $\mathscr{L}^{d}$-measurable set having finite Lebesgue measure, let $g_{A}$ be its covariogram, and let $u \in S^{d-1}$. The following assertions are equivalent:

(i) A has finite directional variation $V_{u}(A)$.

(ii) $\lim _{r \rightarrow 0} \frac{g_{A}(0)-g_{A}(r u)}{|r|}$ exists and is finite.

(iii) The one-dimensional restriction of the covariogram $g_{A}^{u}: r \mapsto g_{A}(r u)$ is Lipschitz.

In addition,

$$
\operatorname{Lip}\left(g_{A}^{u}\right)=\lim _{r \rightarrow 0} \frac{g_{A}(0)-g_{A}(r u)}{|r|}=\frac{1}{2} V_{u}(A),
$$

the second equality being also valid when $V_{u}(A)=$ $+\infty$. 
Remark. Note that Assertion (ii) of Theorem 13 above can be replaced by "The right directional derivative $g_{A}^{u}(0+)$ exists and is finite" since

$$
\begin{aligned}
g_{A}^{u}(0+) & :=\lim _{r \rightarrow 0+} \frac{g_{A}(r u)-g_{A}(0)}{r} \\
& =-\lim _{r \rightarrow 0} \frac{g_{A}(0)-g_{A}(r u)}{|r|} .
\end{aligned}
$$

Proof. Since from Lemma 12,

$$
\frac{g_{A}(0)-g_{A}(r u)}{|r|}=\frac{1}{2} \int_{\mathbb{R}^{d}} \frac{\left|\mathbb{1}_{A}(x+r u)-\mathbb{1}_{A}(x)\right|}{|r|} \mathrm{d} x,
$$

by applying Proposition 11 with $f=\mathbb{1}_{A}$ one obtains the equivalence of $(i)$ and (ii) as well as the formula $\lim _{r \rightarrow 0} \frac{g_{A}(0)-g_{A}(r u)}{|r|}=\frac{1}{2} V_{u}(A)$.

Let us show that (i) implies (iii). By Proposition 5, for all $r$ and $s \in \mathbb{R}$

$$
\begin{aligned}
\mid g_{A}(r u) & -g_{A}(s u) \mid \leq g_{A}(0)-g_{A}((r-s) u) \\
& =\frac{1}{2} \int_{\mathbb{R}^{d}}\left|\mathbb{1}_{A}(x+(r-s) u)-\mathbb{1}_{A}(x)\right| \mathrm{d} x .
\end{aligned}
$$

Applying the inequality of Proposition 11 with $f=\mathbb{1}_{A}$,

$$
\begin{aligned}
& \left|g_{A}(r u)-g_{A}(s u)\right| \\
& \leq \frac{1}{2}|r-s| \int_{\mathbb{R}^{d}} \frac{\left|\mathbb{1}_{A}(x+(r-s) u)-\mathbb{1}_{A}(x)\right|}{|r-s|} \mathrm{d} x \\
& \leq \frac{1}{2} V_{u}(A)|r-s| .
\end{aligned}
$$

Hence $g_{A}^{u}$ is Lipschitz and $\operatorname{Lip}\left(g_{A}^{u}\right) \leq \frac{1}{2} V_{u}(A)$.

Let us now show that (iii) implies (i). For all $r \neq 0$ we have

$$
\begin{aligned}
\operatorname{Lip}\left(g_{A}^{u}\right) \geq & \frac{g_{A}(0)-g_{A}(r u)}{|r|} \\
& =\frac{1}{2} \int_{\mathbb{R}^{d}} \frac{\left|\mathbb{1}_{A}(x+r u)-\mathbb{1}_{A}(x)\right|}{|r|} \mathrm{d} x .
\end{aligned}
$$

By Proposition 11 the right-hand side tends towards $\frac{1}{2} V_{u}(A)$ as $r$ tends to 0 . Hence $A$ has finite directional variation in the direction $u$ and $\operatorname{Lip}\left(g_{A}^{u}\right) \geq \frac{1}{2} V_{u}(A)$. All in all we have shown that $(i)$ and (iii) are equivalent and that $\operatorname{Lip}\left(g_{A}^{u}\right)=\frac{1}{2} V_{u}(A)$.

Considering all the possible directions $u \in S^{d-1}$, the results of the previous theorem lead to Eq. 2 (reproduced below as Eq. 6) and a characterization of sets of finite perimeter.
Theorem 14 (Perimeter and covariogram of measurable sets). Let $A$ be a $\mathscr{L}^{d}$-measurable set having finite Lebesgue measure, and let $g_{A}$ be its covariogram. The following assertions are equivalent:

(i) A has finite perimeter $\operatorname{Per}(A)$.

(ii) For all $u \in S^{d-1}, \quad\left(g_{A}^{u}\right)^{\prime}(0+):=$ $\lim _{r \rightarrow 0+} \frac{g_{A}(r u)-g_{A}(0)}{r}$ exists and is finite.

(iii) The covariogram $g_{A}$ is Lipschitz.

In addition the following relations hold:

$$
\operatorname{Lip}\left(g_{A}\right)=\frac{1}{2} \sup _{u \in S^{d-1}} V_{u}(A) \leq \frac{1}{2} \operatorname{Per}(A)
$$

and

$$
\operatorname{Per}(A)=-\frac{1}{\omega_{d-1}} \int_{S^{d-1}}\left(g_{A}^{u}\right)^{\prime}(0+) \mathscr{H}^{d-1}(\mathrm{~d} u),
$$

this last formula being also valid when $\operatorname{Per}(A)=+\infty$.

Proof. The equivalence of (i) and (ii) as well as the integral geometric formula of Eq. 6 derive from Proposition 8 and the identity

$$
\left(g_{A}^{u}\right)^{\prime}(0+)=\lim _{r \rightarrow 0+} \frac{g_{A}(r u)-g_{A}(0)}{r}=-\frac{1}{2} V_{u}(A) .
$$

Let us now show that (i) implies (iii). Let $y, z \in \mathbb{R}^{d}$. Denote by $u$ the direction of $S^{d-1}$ such that $y-z=$ $|y-z| u$. By Proposition 5 and Theorem 13

$$
\begin{aligned}
& \left|g_{A}(y)-g_{A}(z)\right| \leq g_{A}(0)-g_{A}(y-z) \\
& \quad \leq \frac{1}{2} V_{u}(A)|y-z| \leq\left(\frac{1}{2} \sup _{u \in S^{d-1}} V_{u}(A)\right)|y-z| .
\end{aligned}
$$

Hence $g_{A}$ is Lipschitz and $\operatorname{Lip}\left(g_{A}\right) \leq \frac{1}{2} \sup _{u} V_{u}(A)$. As for the converse implication and inequality, for all $u \in S^{d-1}$,

$$
\operatorname{Lip}\left(g_{A}\right) \geq \lim _{r \rightarrow 0} \frac{g_{A}(0)-g_{A}(r u)}{|r|}=\frac{1}{2} V_{u}(A) .
$$

Hence for all $u \in S^{d-1}, V_{u}(A)<+\infty$ and $\operatorname{Lip}\left(g_{A}\right) \geq$ $\frac{1}{2} \sup _{u} V_{u}(A)$. This concludes the proof.

One natural question is whether Eq. 6 extends to the case of functions, that is if one can recover the variation $V(f)$ of a function $f$ from the directional derivatives of its covariogram $g_{f}(y):=\int_{\mathbb{R}^{d}} f(x+$ $y) f(x) \mathrm{d} x$. The answer to this question is negative. Indeed, if one considers a smooth function $f \in$ $\mathscr{C}_{c}^{1}\left(\mathbb{R}^{d}\right)$, then its covariogram $g_{f}$ is well-defined and is differentiable in 0 . But since $g_{f}$ is even, its derivative at the origin equals zero, and thus the variation of $f$ is not equal to the integral of the directional derivatives of the covariogram $g_{f}$. 


\section{APPLICATIONS TO RANDOM CLOSED SETS}

\section{MEAN COVARIOGRAM OF A RANDOM CLOSED SET}

A random closed set (RACS) $X$ is a measurable map from a probability space $(\Omega, \mathscr{A}, \mathbb{P})$ to the space $\mathscr{F}\left(\mathbb{R}^{d}\right)$ of closed subsets of $\mathbb{R}^{d}$ endowed with the $\sigma$-algebra generated by the family $\left\{\left\{F \in \mathscr{F}\left(\mathbb{R}^{d}\right), F \cap K=\emptyset\right\}, K\right.$ compact $\}$ (Matheron, 1975; Molchanov, 2005; Stoyan et al., 1995).

Definition 15 (Mean covariogram of a random closed set). Let $X$ be a random closed set (RACS) of $\mathbb{R}^{d}$ having finite mean Lebesgue measure, i.e. $\mathbb{E}\left(\mathscr{L}^{d}(X)\right)<+\infty$. The mean covariogram $\gamma_{X}$ of $X$ is the expectation of the covariogram of $X$ with respect to its distribution, that is $\gamma_{X}: \mathbb{R}^{d} \rightarrow[0, \infty)$ is the function defined by

$$
\begin{aligned}
\gamma_{X}(y) & =\mathbb{E}\left(g_{X}(y)\right) \\
& =\mathbb{E}\left(\mathscr{L}^{d}(X \cap(y+X))\right) \\
& =\int_{\mathscr{F}\left(\mathbb{R}^{d}\right)} \mathscr{L}^{d}(A \cap(y+A)) \mathbb{P}_{X}(\mathrm{~d} A) .
\end{aligned}
$$

As the next proposition will show, all the results relative to covariograms of deterministic measurable sets can be adapted for mean covariograms of RACS. However before stating these results, we need to introduce the notions of mean perimeter $\mathbb{E}(\operatorname{Per}(X))$ and mean directional variations $\mathbb{E}\left(V_{u}(X)\right), u \in S^{d-1}$, of a $\operatorname{RACS} X$.

We say that a jointly measurable random field $f: \Omega \times \mathbb{R}^{d} \rightarrow \mathbb{R}$ almost surely (a.s.) in $L_{\mathrm{loc}}^{1}\left(\mathbb{R}^{d}\right)$ has a.s. locally bounded variation if there exists a random $\mathbb{R}^{d}$-valued Radon measure ${ }^{3} D f$ which represents the distributional derivative of $f$, i.e., Eq. 4 holds a.s. If in addition $f \in L^{1}\left(\mathbb{R}^{d}\right)$ a.s. and $|D f|\left(\mathbb{R}^{d}\right)$ is a.s. finite, then $f$ is said to have a.s. bounded variation in $\mathbb{R}^{d}$. Similarly, $f: \Omega \times \mathbb{R}^{d} \rightarrow \mathbb{R}$ a.s. in $L_{\text {loc }}^{1}\left(\mathbb{R}^{d}\right)$ has a.s. locally bounded directional variation in the direction $u \in S^{d-1}$ if there exists a random signed Radon measure $D_{u} f$ representing the distributional directional derivative of $f$. If $f \in L^{1}\left(\mathbb{R}^{d}\right)$ a.s. and $\left|D_{u} f\right|\left(\mathbb{R}^{d}\right)<+\infty$ a.s., then one says that $f$ has bounded directional variation in the direction $u$.

The mean variation $\mathbb{E}(V(f))$ and the mean directional variations $\mathbb{E}\left(V_{u}(f)\right), u \in S^{d-1}$, of a random field $f$ a.s. in $L^{1}\left(\mathbb{R}^{d}\right)$ are defined by

$$
\mathbb{E}(V(f))= \begin{cases}\mathbb{E}\left(|D f|\left(\mathbb{R}^{d}\right)\right) & \text { if } f \text { has a.s. bounded } \\ +\infty & \text { variation, } \\ \text { otherwise }\end{cases}
$$

and

$$
\mathbb{E}\left(V_{u}(f)\right)= \begin{cases}\mathbb{E}\left(\left|D_{u} f\right|\left(\mathbb{R}^{d}\right)\right) & \text { if } f \text { has a.s. bounded } \\ & \text { directional variation, } \\ +\infty & \text { otherwise. }\end{cases}
$$

Since any RACS $X$ defines a jointly measurable random field by $(\omega, x) \mapsto \mathbb{1}_{X(\omega)}(x)$ (see (Molchanov, 2005 , p. 59)), the mean perimeter $\mathbb{E}(\operatorname{Per}(X))$ and the mean directional variations $\mathbb{E}\left(V_{u}(X)\right)$ of a RACS $X$ are well-defined.

Proposition 16 (Properties of the mean covariogram of a RACS). Let $X$ be a RACS of $\mathbb{R}^{d}$ satisfying $\mathbb{E}\left(\mathscr{L}^{d}(X)\right)<+\infty$ and let $\gamma_{X}$ be its mean covariogram. Then

1. For all $y \in \mathbb{R}^{d}, 0 \leq \gamma_{X}(y) \leq \gamma_{X}(0)=\mathbb{E}\left(\mathscr{L}^{d}(X)\right)$.

2. $\gamma_{X}$ is even.

3. $\gamma_{X}(y)=\int_{\mathbb{R}^{d}} \mathbb{P}(x \in X$ and $x+y \in X) \mathrm{d} x$.

4. $\int_{\mathbb{R}^{d}} \gamma_{X}(y) d y=\mathbb{E}\left(\mathscr{L}^{d}(X)^{2}\right) \in[0,+\infty]$.

5. If $\mathbb{E}\left(\mathscr{L}^{d}(X)\right)>0$, then $\gamma_{X}$ is a strictly positivedefinite function.

6. For all $y, z \in \mathbb{R}^{d}$,

$$
\left|\gamma_{X}(y)-\gamma_{X}(z)\right| \leq \gamma_{X}(0)-\gamma_{X}(y-z) .
$$

7. $\gamma_{X}$ is uniformly continuous over $\mathbb{R}^{d}$ and $\lim _{|y| \rightarrow+\infty} \gamma_{X}(y)=0$.

8. We have

$$
\begin{gathered}
\lim _{r \rightarrow 0} \frac{\gamma_{X}(0)-\gamma_{X}(r u)}{|r|}=\frac{1}{2} \mathbb{E}\left(V_{u}(X)\right) \\
\text { and, noting }\left(\gamma_{X}^{u}\right)^{\prime}(0+)=\lim _{r \rightarrow 0+} \frac{\gamma_{X}(r u)-\gamma_{X}(0)}{r} \\
-\frac{1}{\omega_{d-1}} \int_{S^{d-1}}\left(\gamma_{X}^{u}\right)^{\prime}(0+) \mathscr{H}^{d-1}(\mathrm{~d} u)=\mathbb{E}(\operatorname{Per}(X)) .
\end{gathered}
$$

The proofs are omitted since they mostly consist in integrating the results of the previous sections with respect to the distribution of the RACS $X$. When $\mathbb{E}\left(V_{u}(X)\right)<+\infty$ and $\mathbb{E}(\operatorname{Per}(X))<+\infty$, both formulas of property 8 follows easily from the bounded convergence theorem. Using Fatou's lemma, one shows that these formulas are also valid when $\mathbb{E}\left(V_{u}(X)\right)=+\infty$ and $\mathbb{E}(\operatorname{Per}(X))=+\infty$.

\footnotetext{
${ }^{3}$ We refer to (Horowitz, 1985) for definitions relative to random Radon measures and for a proof of the fact that the variation $|\mu|$ of a random Radon measure $\mu$ is a well-defined random positive Radon measure.
} 


\section{SPECIFIC VARIATION OF A STATIONARY RACS}

A RACS $X$ is said to be stationary if for all $y \in \mathbb{R}^{d}$, the translated RACS $y+X$ has the same distribution as $X$. If a $\operatorname{RACS} X$ is stationary, one defines its variogram $v_{X}$ as the function $v_{X}(y)=$ $\mathbb{P}(y \in X, 0 \notin X)$ (see e.g., (Lantuéjoul, 2002) for more details on variograms).

If a stationary RACS $X$ has locally bounded variation, then one easily checks that its derivative $D \mathbb{1}_{X}$, which is by definition a random $\mathbb{R}^{d}$-valued Radon measure, is also stationary. Consequently, the variation measure $\left|D \mathbb{1}_{X}\right|$ is a stationary positive Radon measure, and thus there exists a real number $\theta_{V}(X) \in$ $[0,+\infty]$ such that for all nonempty open sets $U \subset \subset \mathbb{R}^{d}$,

$$
\mathbb{E}(V(X, U)):=\mathbb{E}\left(\left|D \mathbb{1}_{X}\right|(U)\right)=\theta_{V}(X) \mathscr{L}^{d}(U) .
$$

We choose to call this constant $\theta_{V}(X)$ the specific variation of $X$ or the variation intensity of $X$ (see the discussion below). Similarly, for all $u \in$ $S^{d-1}$ there exists a real $\theta_{V_{u}}(X) \in[0,+\infty]$ such that $\mathbb{E}\left(\left|D_{u} \mathbb{1}_{X}\right|(U)\right)=\theta_{V_{u}}(X) \mathscr{L}^{d}(U) . \theta_{V_{u}}(X)$ is called the specific directional variation of $X$ in the direction $u$ (or also the directional variation intensity). As before, one extends the definition of the specific variation for stationary $\operatorname{RACS} X$ which do not have a.s. locally bounded variation by setting $\theta_{V}(X)=+\infty$, and similarly for the specific directional variation $\theta_{V_{u}}(X)$. In this context the integral-geometric formula of Eq. 5 gives

$$
\theta_{V}(X)=\frac{1}{2 \omega_{d-1}} \int_{S^{d-1}} \theta_{V_{u}}(X) \mathscr{H}^{d-1}(\mathrm{~d} u) .
$$

Theorem 17 (Specific variations and variogram). Let $X$ be a stationary $R A C S$, let $v_{X}$ be its variogram, and, for all $u \in S^{d-1}$, denote $\left(v_{X}^{u}\right)^{\prime}(0+):=\lim _{r \rightarrow 0+} \frac{1}{r} v_{X}(r u)$. Then for all $u \in S^{d-1}$ the specific directional variation $\theta_{V_{u}}(X)$ is given by

$$
\theta_{V_{u}}(X)=2\left(v_{X}^{u}\right)^{\prime}(0+)=2 \lim _{r \rightarrow 0} \frac{1}{|r|} \mathbb{P}(r u \in X, 0 \notin X) .
$$

In other words, the specific directional variation is twice the right directional derivative of the variogram at the origin. Integrating over all directions, one obtains the specific variation of $X$ :

$$
\theta_{V}(X)=\frac{1}{\omega_{d-1}} \int_{S^{d-1}}\left(v_{X}^{u}\right)^{\prime}(0+) \mathscr{H}^{d-1}(\mathrm{~d} u) .
$$

Before proving this theorem let us discuss the terminology specific variation of $X$ for the constant
$\theta_{V}(X)$. Eq. 7 is the formula given in (Lantuéjoul, 2002, p. 26) and which originates from Matheron (1967, p. 30). In these references, the constant corresponding to the variation intensity $\theta_{V}(X)$ is called the specific $(d-1)$-volume of $X$ (specific perimeter if $d=2$, specific surface area if $d=3$ ). However, in the later works of Matheron (1975) as well as on recent reference textbooks (Stoyan et al., 1995; Schneider and Weil, 2008), the specific surface measure refers to the surface measure that derives from Steiner's formula. This measure has different names, depending on its normalization and the degree of generalization: intrinsic volume of index $d-1$ and Minkowski's content of index 1 for convex sets (Schneider and Weil, 2008), total curvature of index $d-1$ for sets with positive reach and $\mathscr{U}_{P R}$-sets (Federer, 1959; Rataj and Zähle, 2001), or also, in a more general setting, outer Minkowski content (Ambrosio et al., 2008; Villa, 2009; see also Hug et al., 2004). Even though the (variational) perimeter of a set and this notion of surface measure agree for convex sets (Ambrosio et al., 2008), the distinction is important. Indeed their extensions to non convex sets have different behaviors. For example, the outer Minkowski content counts twice the isolated fine parts of a set having a bounded and $(d-1)$-rectifiable topological boundary, whereas these fine parts have no influence on the perimeter (Villa, 2009, Proposition 4.1) (here "isolated fine parts" denotes the part of the boundary which has Lebesgue density 0 ). In order to make a clear distinction between the (variational) perimeter and the surface measure from Steiner's formula, the constant $\theta_{V}(X)$ is named the specific variation of $X$ and not its "specific perimeter".

As mentioned in the introduction, one should notice that, contrary to the specific surface area (Schneider and Weil, 2008), the specific variation $\theta_{V}(X)$ is well-defined for any stationary RACS. Besides, Theorem 17 shows that the specific directional variations $\theta_{V_{u}}(X)$ and the specific variation $\theta_{V}(X)$ are easily computed as soon as one knows the variogram of $X$. This will be illustrated in the next section where the specific variations of stationary Boolean models are computed.

Let us now turn to the proof of Theorem 17 which uses the following intuitive lemma.

Lemma 18. Let $A$ be a $\mathscr{L}^{d}$-measurable set and $B$ be an open ball. Then for all $u \in S^{d-1}$,

$$
V_{u}(A, B) \leq V_{u}\left(A \cap B, \mathbb{R}^{d}\right) \leq V_{u}(A, B)+V_{u}\left(B, \mathbb{R}^{d}\right) .
$$

References for the proof. The first inequality is immediate from the definition of the directional 
variation on an open set (Ambrosio et al., 2000). The second inequality is easily proved using the interpretation of the directional variation as the projection measure of the essential boundary mentioned in the introduction (Chlebík, 1997).

Proof of Theorem 17. First remark that

$$
\begin{aligned}
\mathbb{P}(r u \in X, 0 \notin X)=\mathbb{P}(0 \in X,-r u \notin X) \\
\quad=\mathbb{P}(0 \in X)-\mathbb{P}(0 \in X \text { and }-r u \in X) \\
\quad=\mathbb{P}(0 \in X)-\mathbb{P}(0 \in X \cap(r u+X)) .
\end{aligned}
$$

Let $B$ be any nonempty open ball. Since $X$ is a stationary RACS

$$
\mathbb{P}(0 \in X)=\frac{\mathbb{E}\left(\mathscr{L}^{d}(X \cap B)\right)}{\mathscr{L}^{d}(B)} .
$$

As $X \cap(r u+X)$ is also a stationary RACS, we have

$$
\mathbb{P}(0 \in X \cap(r u+X))=\frac{\mathbb{E}\left(\mathscr{L}^{d}(X \cap(r u+X) \cap B)\right)}{\mathscr{L}^{d}(B)} .
$$

In order to introduce the mean covariogram of the set $X \cap B$, let us denote $E_{r}=(X \cap B) \cap(r u+(X \cap B))$. Clearly we have the following inclusions

$$
E_{r} \subset X \cap(r u+X) \cap B
$$

and

$$
[X \cap(r u+X) \cap B] \backslash E_{r} \subset B \backslash(B \cap(r u+B)) .
$$

Noting that $\mathscr{L}^{d}(B \backslash(B \cap(r u+B)))=g_{B}(0)-g_{B}(r u)$, we obtain

$$
\begin{aligned}
\frac{\gamma_{X \cap B}(r u)}{\mathscr{L}^{d}(B)} & \leq \frac{\mathbb{E}\left(\mathscr{L}^{d}(X \cap(r u+X) \cap B)\right)}{\mathscr{L}^{d}(B)} \\
& \leq \frac{\gamma_{X \cap B}(r u)}{\mathscr{L}^{d}(B)}+\frac{g_{B}(0)-g_{B}(r u)}{\mathscr{L}^{d}(B)} .
\end{aligned}
$$

This yields both an upper and a lower bound of $\mathbb{P}(r u \in X, 0 \notin X)$. We have

$$
\mathbb{P}(r u \in X, 0 \notin X) \leq \frac{\gamma_{X \cap B}(0)-\gamma_{X \cap B}(r u)}{\mathscr{L}^{d}(B)} .
$$

By property 8 of Proposition 16 and the second inequality of Lemma 18 ,

$$
\begin{aligned}
\limsup _{r \rightarrow 0} \frac{1}{|r|} \mathbb{P}(r u \in X, 0 \notin X) & \leq \frac{1}{2} \frac{\mathbb{E}\left(V_{u}(X \cap B)\right)}{\mathscr{L}^{d}(B)} \\
& \leq \frac{1}{2} \theta_{V_{u}}(X)+\frac{1}{2} \frac{V_{u}(B)}{\mathscr{L}^{d}(B)} .
\end{aligned}
$$

As for the lower bound,

$$
\begin{aligned}
& \mathbb{P}(r u \in X, 0 \notin X) \\
& \geq \frac{\gamma_{X \cap B}(0)-\gamma_{X \cap B}(r u)}{\mathscr{L}^{d}(B)}-\frac{g_{B}(0)-g_{B}(r u)}{\mathscr{L}^{d}(B)} .
\end{aligned}
$$

Again, by Proposition 16 and the first inequality of Lemma 18, we have

$$
\begin{aligned}
& \liminf _{r \rightarrow 0} \frac{1}{|r|} \mathbb{P}(r u \in X, 0 \notin X) \\
& \geq \frac{1}{2} \frac{\mathbb{E}\left(V_{u}(X \cap B)\right)}{\mathscr{L}^{d}(B)}-\frac{1}{2} \frac{V_{u}(B)}{\mathscr{L}^{d}(B)} \\
& \geq \frac{1}{2} \theta_{V_{u}}(X)-\frac{1}{2} \frac{V_{u}(B)}{\mathscr{L}^{d}(B)} .
\end{aligned}
$$

The two established inequalities are true for any nonempty open ball $B$. Noting $R$ the radius of $B$,

$$
\frac{V_{u}(B)}{\mathscr{L}^{d}(B)}=\frac{\omega_{d-1} R^{d-1}}{\omega_{d} R^{d}}=\frac{\omega_{d-1}}{\omega_{d}} \frac{1}{R} .
$$

The enunciated formula is obtained by letting $R$ tends to $+\infty$.

\section{COMPUTATION OF THE SPECIFIC VARIATIONS OF BOOLEAN MODELS}

In this section we apply Theorem 17 to compute the specific directional variations and the specific variation of any stationary Boolean model. The Boolean model (Stoyan et al., 1995; Schneider and Weil, 2008) with intensity $\lambda$ and grain distribution $P_{X}$ is the stationary RACS $Z$ defined by

$$
Z=\bigcup_{i \in \mathbb{N}} x_{i}+X_{i}
$$

where $\left\{x_{i}, i \in \mathbb{N}\right\} \subset \mathbb{R}^{d}$ is a stationary Poisson point process with intensity $\lambda>0$ and $\left(X_{i}\right)_{i \in \mathbb{N}}$ is a sequence of i.i.d. RACS with common distribution $P_{X}$, independent of $\left\{x_{i}, i \in \mathbb{N}\right\}$. Moreover, the RACS $\left(X_{i}\right)$ are supposed to have a finite mean Lebesgue measure (otherwise $Z=\mathbb{R}^{d}$ a.s.). The avoiding functional of the Boolean model $Z$ is well-known: for any compact $K \subset \mathbb{R}^{d}$ we have

$$
\mathbb{P}(Z \cap K=\emptyset)=\exp \left(-\lambda \mathbb{E}\left(\mathscr{L}^{d}(X \oplus \check{K})\right)\right),
$$

where $X$ denotes a RACS with distribution $P_{X}$ and $X \oplus \breve{K}=\{x-y, x \in X, y \in K\}$ (see, e.g., Stoyan et al., 1995, p. 65 or Lantuéjoul, 2002, p. 164). Starting from the general expression of Eq. 8 (which determines the distribution of $Z$ ), let us compute the variogram $v_{Z}$ of $Z$. For $K=\{0\}$, Eq. 8 becomes

$$
q:=\mathbb{P}(0 \notin Z)=\exp \left(-\lambda \mathbb{E}\left(\mathscr{L}^{d}(X)\right)\right) .
$$


For $K=\{0,-r u\}$, with $r \neq 0$ and $u \in S^{d-1}$, remark that we have

$$
\begin{aligned}
\mathscr{L}^{d}(X \oplus \check{K}) & =\mathscr{L}^{d}(X \cup r u+X) \\
& =2 \mathscr{L}^{d}(X)-\mathscr{L}^{d}(X \cap(r u+X)) .
\end{aligned}
$$

Hence in this case

$$
\mathbb{E}\left(\mathscr{L}^{d}(X \oplus \check{K})\right)=2 \mathbb{E}\left(\mathscr{L}^{d}(X)\right)-\gamma_{X}(r u) .
$$

As a result the variogram $v_{Z}$ is equal to (Stoyan et al., 1995, p. 68; Lantuéjoul, 2002, p. 165)

$$
\begin{aligned}
v_{Z}(r u) & =\mathbb{P}(-r u \in Z \text { and } 0 \notin Z) \\
& =\mathbb{P}(0 \notin Z)-\mathbb{P}(Z \cap\{0,-r u\}=\emptyset) \\
& =q-\exp \left(-\lambda\left(2 \mathbb{E}\left(\mathscr{L}^{d}(X)\right)-\gamma_{X}(r u)\right)\right) \\
& =q-q \exp \left(-\lambda\left(\gamma_{X}(0)-\gamma_{X}(r u)\right)\right) .
\end{aligned}
$$

By Theorem 17 and property 8 of Proposition 16 we deduce

$$
\begin{aligned}
\theta_{V_{u}}(Z) & =2\left(v_{Z}^{u}\right)^{\prime}(0+) \\
& =2 q \lambda \frac{1}{2} \mathbb{E}\left(V_{u}(X)\right) \\
& =\lambda \mathbb{E}\left(V_{u}(X)\right) \exp \left(-\lambda \mathbb{E}\left(\mathscr{L}^{d}(X)\right)\right) .
\end{aligned}
$$

Integrating this formula over all directions $u$ we obtain $\theta_{V}(Z)$. Our computation is summarized in the following statement.

Proposition 19 (Specific variations of a stationary Boolean model). Let $Z$ be the Boolean model with Poisson intensity $\lambda$ and grain distribution $P_{X}$, let $X$ be a RACS with distribution $P_{X}$, and suppose that $\mathbb{E}\left(\mathscr{L}^{d}(X)\right)<+\infty$. Then for all $u \in S^{d-1}$,

$$
\theta_{V_{u}}(Z)=\lambda \mathbb{E}\left(V_{u}(X)\right) \exp \left(-\lambda \mathbb{E}\left(\mathscr{L}^{d}(X)\right)\right)
$$

and

$$
\theta_{V}(Z)=\lambda \mathbb{E}(\operatorname{Per}(X)) \exp \left(-\lambda \mathbb{E}\left(\mathscr{L}^{d}(X)\right)\right)
$$

Eq. 9 is valid for any grain distribution $P_{X}$ and generalizes known results for Boolean models with convex grains (Schneider and Weil, 2008, p. 386). Similar generalizations involving intensity of surface measures deriving from Steiner's formula have recently been established (Hug et al., 2004; Villa, 2010). As already stressed out, our result is similar but not identical since the outer Minkowski content of a set differs from its (variational) perimeter (Villa, 2009).

A promising direction for further works is to extend the notion of specific variation for non stationary RACS. In particular, following (Villa, 2010), one could try to derive local variation densities of certain non stationary Boolean models.

\section{REFERENCES}

Ambrosio L, Colesanti A, Villa E (2008). Outer Minkowski content for some classes of closed sets. Math Ann 342:727-48.

Ambrosio L, Fusco N, Pallara D (2000). Functions of bounded variation and free discontinuity problems. Oxford: Oxford University Press.

Averkov G, Bianchi G (2009). Confirmation of Matheron's conjecture on the covariogram of a planar convex body. J Eur Math Soc 11:1187-202.

Bordenave C, Gousseau Y, Roueff F (2006). The dead leaves model: a general tessellation modeling occlusion. Adv Appl Prob 38:31-46.

Cabo A, Baddeley A (1995). Line transects, covariance functions and set convergence. Adv Appl Prob 7:585605.

Cabo AJ, Janssen RHP (1994). Cross-covariance functions characterise bounded regular closed sets. Tech. Rep. BS-R9426, CWI.

Chlebík M (1997). On variation of sets. preprint 44, Max-Planck-Institut für Mathematik in den Naturwissenschaften Leipzig.

Federer H (1959). Curvature measures. Trans Amer Math Soc 93:418-91.

Haas A, Matheron G, Serra J (1967). Morphologie mathématique et granulométries en place. Annales des Mines .

Heinrich L, Schmidt V (1985). Normal convergence of multidimensional shot noise and rates of this convergence. Adv Appl Prob 17:709-30.

Hirsch F, Lacombe G (1999). Elements of functional analysis, vol. 192 of Graduate texts in mathematics. New York: Springer.

Horowitz J (1985). Measure-valued random processes. Probab Theory Rel 70:213-36.

Hug D, Last G, Weil W (2004). A local Steiner-type formula for general closed sets and applications. Math Z 246:237-72.

Jeulin D (1997). Dead leaves models: from space tesselation to random functions. In: Jeulin D, ed., Proc. of the Symposium on the Advances in the Theory and Applications of Random Sets. World Scientific Publishing Company.

Lantuéjoul C (2002). Geostatistical simulation: models and algorithms. Berlin: Springer.

Matheron G (1965). Les variables régionalisées et leur estimation. Paris: Masson.

Matheron G (1967). Éléments pour une théorie des mileux poreux. Paris: Masson. 
Matheron G (1968). Schéma booléen séquentiel de partition aléatoire. Tech. Rep. 89, CMM.

Matheron G (1975). Random sets and integral geometry. Wiley series in probability and mathematical statistics. John Wiley \& Sons.

Matheron G (1986). Le covariogramme géométrique des compacts convexes de $\mathbb{R}^{2}$. Tech. Rep. N/2/86/G, Centre de Géostatistique, Ecole des mines de Paris.

Molchanov I (2005). Theory of Random Sets. Probability and Its Applications. Springer.

Rataj J (2004). On set covariance and three-point test sets. Czechoslovak Math 54(129):205-14.

Rataj J, Zähle M (2001). Curvatures and currents for unions of sets with positive reach, II. Ann Glob Anal Geom 20:1-21.
Rice J (1977). On generalized shot noise. Adv Appl Prob 9:553-65.

Schneider R, Weil W (2008). Stochastic and Integral Geometry. Probability and Its Applications. Springer.

Serra J (1982). Image Analysis and Mathematical Morphology, vol. 1. London: Academic press.

Stoyan D, Kendall WS, Mecke J (1995). Stochastic geometry and its applications. Wiley series in probability and mathematical statistics. John Wiley \& Sons, 2nd ed.

Villa E (2009). On the outer Minkowski content of sets. Ann Mat Pura Appl 188:619-30.

Villa E (2010). Mean densities and spherical contact distribution function of inhomogeneous Boolean models. Stoch Anal Appl 28:480-504. 